\section{Impact and cost of algorithms for the diagnosis of adults with pulmonary tuberculosis in South Africa}

To the Editor: We wish to respond to the letter ${ }^{[1]}$ commenting on our article 'Diagnosing Xpert MTB/RIF-negative TB: Impact and cost of alternative algorithms for South Africa. ${ }^{[2]}$

The National TB Cost Model was established to assist the South African Department of Health in understanding both the costs and the impact of alternative algorithms for the diagnosis of adults with symptoms of pulmonary tuberculosis (PTB) presenting for ambulatory care, with impact measured as the number of drugsensitive and drug-resistant TB cases diagnosed and treated. ${ }^{[3]}$ The analysis in the $S A M J^{[2]}$ addressed one arm of this algorithm, that of patients with symptoms suggestive of PTB who are HIV-infected but have a negative Xpert MTB/RIF test. Patients who are unable to produce sputum for testing (often because of young age) or have symptoms of extrapulmonary TB (EPTB) are not included in the Xpert-based algorithm adopted in South Africa $(\mathrm{SA})^{[4]}$ and were therefore not included in our analysis of alternatives. We agree, however, that modelling the impact and cost of alternative algorithms for diagnosis of paediatric TB and EPTB will be essential in guiding policy decisions as these algorithms are further developed.

Black $^{[1]}$ concludes that 'testing large numbers of patients with a second GXP for a $<3 \%$ positive rate is not economically prudent', a strong statement that is not referenced or supported in the text of the letter. In fact, our analysis concluded that, given SA's TB diagnostic guideline to continue to investigate patients who are HIV-infected and Xpert-negative for PTB despite the low yield of TB cases, a cost saving could be achieved by implementing a second Xpert MTB/RIF test instead of the smear, chest X-ray, culture and Hain line-probe assay (for positive cultures) currently required. In addition to the money saved, the alternative algorithm using two Xpert tests could potentially increase the proportion of patients actually treated, with subsequent reductions in risk of transmission and improvements in treatment outcomes, as Xpert results are available much more rapidly than culture results.

The TB diagnostic algorithm is a tool to assist those who diagnose $\mathrm{TB}$ most often in SA - nurses at primary healthcare clinics. Guidelines, including algorithms, are there to support good clinical practice, not to be implemented blindly. Our modelling analysis suggests that an algorithm for adult, ambulatory PTB that calls for a second Xpert test for those who are HIV-infected will be less costly and result in more patients on treatment than the current culture-based algorithm. Other algorithms must still be developed for EPTB, paediatric TB, and non-TB conditions with similar symptoms.

\section{Kathryn Schnippel}

Lawrence Long

Health Economics and Epidemiology Research Office, Department of Internal Medicine, School of Clinical Medicine, Faculty of Health Sciences, University of the Witwatersrand, Johannesburg, South Africa

kschnippel@heroza.org

\section{Gesine Meyer-Rath}

\section{Ian Sanne}

Sydney Rosen

Center for Global Health and Development, Boston University, Boston, USA; and Health Economics and Epidemiology Research Office, Department of Internal Medicine, School of Clinical Medicine, Faculty of Health Sciences, University of the Witwatersrand, Johannesburg, South Africa

\section{Wendy Stevens}

Department of Molecular Medicine and Haematology, Faculty of Health Sciences, University of the Witwatersrand; and National Health Laboratory Service, Johannesburg, South Africa

\footnotetext{
1. Black A. A new algorithm for the diagnosis of all forms of tuberculosis is required for South Africa. S Afr Med J 2013;103(6):355-356. [http://dx.doi.org/10.7196/SAMJ.6896]

Schnippel K, Meyer-Rath G, Long L, Stevens WS, Sanne I, Rosen S. Diagnosing Xpert MTB/RIF-negative TB: Impact and cost of alternative algorithms for South Africa. S Afr Med J 2013;103(2):101-106. [http://
. TB: Impact and cost of alternative
dx.doi.org/10.7196/SAMJ.6182]

3. Meyer-Rath G, Schnippel K, Long L, et al. The impact and cost of scaling up GeneXpert MTB/RIF in South Africa. PLoS ONE 2012;7(5):e36966. [http://dx.doi.org/10.1371/journal.pone.0036966]

4. Directorate Drug-Resistant TB, TB \& HIV. Management of Drug-Resistant Tuberculosis: Policy Guidelines. Pretoria: Government Printer, 2011. http://www.doh.gov.za/docs/policy/2012/TBpolicy.pdf
(accessed 15 March 2013).
}

S Afr Med J 2013;103(7):436. DOI:10.7196/SAMJ.7156 